\title{
Dentigerous cysts associated with impacted supernumerary teeth in the anterior maxilla
}

\author{
QIAN JIANG, GUANG-ZHOU XU, CHI YANG, CHUANG-QI YU, DONG-MEI HE and ZHI-YUAN ZHANG \\ Department of Oral and Maxillofacial Surgery, Ninth People's Hospital, College of Stomatology, \\ Shanghai Jiao Tong University School of Medicine, Shanghai Research Institute of Stomatology, \\ Shanghai Key Laboratory of Stomatology, Shanghai 200011, P.R. China
}

Received February 3, 2011; Accepted April 3, 2011

DOI: $10.3892 /$ etm.2011.274

\begin{abstract}
Dentigerous cysts are thought to be caused by a developmental abnormality derived from the reduced enamel epithelium of the tooth forming organ. Most typical dentigerous cysts are those associated with the third molar teeth of the mandible, but rarely involve impacted supernumerary teeth in the anterior maxilla. Swelling and/or pain may be the major complaints of the patients. Herein, we review the literature spanning the past 22 years concerning dentigerous cysts associated with supernumerary teeth in the anterior maxilla, and present four additional cases with emphasis given to the clinicopathological characteristics of this type of dentigerous cyst.
\end{abstract}

\section{Introduction}

Dentigerous cyst, also known as follicular cyst, is an odontogenic cyst caused by fluid accumulation between the reduced enamel epithelium and the enamel surface of a formed tooth. It is thought to be a developmental abnormality derived from the reduced enamel epithelium of the tooth forming organ (1).

A dentigerous cyst is most frequently found in individuals in the age group between 20 and 40 years (2). Most typical dentigerous cysts are those associated with the third molar teeth of the mandible, followed by maxillary third molars, maxillary canines and premolars of both the maxillary and mandibular bones (3). They are occasionally associated with supernumerary teeth $(3,4)$. Stafne first described dentigerous cysts associated with supernumerary teeth and found an incidence of 5.5\% among 200 supernumerary teeth (8). Most supernumerary teeth are noted in the anterior maxillary region.

Correspondence to: Dr Guang-Zhou Xu, Department of Oral and Maxillofacial Surgery, Ninth People's Hospital, College of Stamotology, Shanghai Jiao Tong University School of Medicine, Shanghai Research Institute of Stomatology, Shanghai Key Laboratory of Stomatology, Shanghai 200011, P.R. China

E-mail: xuguangzhou1968@163.com

Key words: dentigerous cyst, supernumerary teeth, mesiodens, anterior maxilla
The most common supernumerary tooth which appears in the maxillary midline has also been named a mesiodens due to its position in the center of the maxilla (6).

In the present study, we review the literature spanning the past 22 years concerning dentigerous cysts associated with supernumerary teeth in the anterior maxilla, and present four additional cases with emphasis on the clinicopathological characteristics of this type of dentigerous cyst. A search of Medline from 1988 to 2010 was conducted, using the key words 'dentigerous cyst', 'mesiodens' and 'supernumerary tooth'.

\section{Case reports}

Case 1. A 55-year-old Chinese female attended our clinic with a chief complaint of painless swelling in the palatine for a duration of 6 months. There was no history of allergic symptoms and systemic illness. On intraoral examination, a soft, fluctuant, painless swelling was palpable in the anterior portion of the hard palatine. There was no history of trauma or no dental treatment around the lesion. The maxillary incisors were sensitive to percussion, but responded positively to vitality tests. The patient's neurological exam was normal. The upper occlusal radiograph of the lesion revealed an impacted supernumerary tooth with a short root apical to the maxillary left central incisor which was associated with a well-defined radiolucent area in the anterior region of the maxilla. It also showed that the pericementum of the maxillary central incisors was continuous and intact, and the apices of the left tooth existed in the cyst. Displacement of the roots of the maxillary central incisors was evident (Fig. 1D). Computed tomography (CT) scan revealed soft tissue density measuring 1.9x1.4 cm in the midline, containing the impacted supernumerary tooth (Fig. 1A-C). A clinical diagnosis of an infected dentigerous cyst associated with a supernumerary tooth (a mesiodens) was made.

After the acute stage subsided, routine blood investigations were normal. With the patient under local anesthesia, a full-thickness mucoperiosteal flap was reflected at the right mucopalatine fold from the maxillary right second premolar tooth to the maxillary left second premolar. A cyst measuring $2 \mathrm{~cm}$ in diameter was enucleated. The cyst consisted of a smooth outer surface and a well-formed wall that was 1-mm thick, surrounding the impacted supernumerary tooth 
(Fig. E). During surgery, there was no connection between the supernumerary tooth and the apices of the maxillary left center incisor. Histological examination of the specimen confirmed the initial diagnosis (Fig. 1F). Postoperative antibiotics were prescribed for 5 days, and the stitches were removed after 1 week. One month later, the patient presented no complications after receiving cyst enucleation.

Case 2. A 46-year-old Chinese male attended our clinic with a chief complaint of painless swelling in the palatine for a duration of one year. There was no history of trauma or no dental treatment around the lesion. Intraoral examination revealed a solitary and well-defined swelling in the right premaxillary region. There was no history of trauma, and vitality of the associated teeth was intact. The radiological examination included panoramic and occlusal radiographs that revealed one supernumerary tooth involved in a solitary, well-defined radiolucency, extending from the midline to the right maxillary lateral region, apically to the right upper central incisor (Fig. 2A and B). CT revealed a supernumerary tooth surrounded by a soft tissue mass measuring $1.6 \times 2.04 \mathrm{~cm}$ in the right premaxillary region, with the palatal cortical expanded. There was no bone destruction (Fig. 2C and D). A tentative diagnosis of dentigerous cyst associated with a supernumerary tooth was made.

Routine blood investigations were normal, and the cyst was enucleated along with the supernumerary tooth under local anesthesia. The macroscopic findings revealed cystic lining attachments to the supernumerary tooth (Fig. 2E). Routine histological examination of the enucleated specimen confirmed the initial diagnosis (Fig. 2F). After surgery, antibiotics were prescribed for 5 days, and the stitches were removed after 1 week. The postoperative period of the patient was uneventful.

Case 3. A 53-year-old Chinese male attended our clinic with a chief complaint of a painless slow-growing mass on the upper lip for a duration of one year. The right upper lateral incisor had been extracted several years earlier. Intraoral examination revealed a soft, fluctuant, labial cyst-like swelling measuring $2.5 \times 1.5 \mathrm{~cm}$ on the right upper lip (Fig. 3A). The maxillary right canine and maxillary right central incisor were vital and not sensitive to percussion. A panoramic radiograph revealed a relatively large and well-defined radiolucency between the maxillary right canine and the right central incisor, enveloping an unerupted horizontal supernumerary tooth (Fig. 3B). CT showed a supernumerary tooth surrounded by a soft tissue mass measuring $2.4 \mathrm{~cm}$ horizontally, $1.5 \mathrm{~cm}$ vertically and $1.3 \mathrm{~cm}$ sagittally, with the labial cortical bone expanded and heavily eroded (Fig. 3C-E). A tentative diagnosis of infected dentigerous cyst associated with a supernumerary tooth was made.

The patient's general health and development were normal except for this lesion. Under local anesthesia, the cyst was wholly enucleated together with the unerupted supernumerary tooth. The extracted specimen consisted of a brown cyst measuring $2.5 \times 0.5 \times 1.0 \mathrm{~cm}$ with a small monoradicular malformed supernumerary tooth (Fig. 3F). Routine histological examination of the enucleated specimen confirmed the initial diagnosis (Fig. 2F). After surgery, antibiotics were prescribed for 5 days, and the stitches were removed after 1 week. The postoperative period of the patient was uneventful.
Case 4. A 23-year-old Chinese male attended our clinic with a chief complaint of painless swelling in the palatine for a duration of one year. There was no history of allergic symptoms and systemic illness. On intraoral examination, a soft, painless swelling was palpable in the anterior portion of the hard palatine. There was no history of trauma or no dental treatment around the lesion. Maxillary anterior teeth were vital and not sensitive to percussion. The radiological examination including panoramic and occlusal radiographs revealed an impacted inverted supernumerary tooth with a short root apical to the maxillary right lateral incisor which was associated with a well-defined radiolucent area extending from the maxillary right first molar to the maxillary right cental incisor. None of the apices of these teeth existed in the cyst (Fig. 4A and B). CT demonstrated that the radiolucent lesion measuring $1.74 \times 1.5 \mathrm{~cm}$ in the right premaxillary region involved an inverted-shaped tooth-like calcified structure (Fig. 4C).

Routine blood investigations were abnormal with a hemoglobin level $<68 \mathrm{~g} / \mathrm{l}$. Surgery could not be performed because of severe anemia.

\section{Discussion}

Supernumerary teeth are commonly located in the anterior maxillary region and can often cause developmental and eruption disturbances of adjacent permanent teeth, leading to crowding, displacement, diastema and, in some cases, radicular resorption and dentigerous cyst formation (6-8). The four cases described the dentigerous cyst formation involving a supernumerary tooth in the anterior maxilla. In addition, displacement of the adjacent central incisors was only observed in case 1 and none of the 4 patients had root resorption.

Dentigerous cysts are the second most common odontogenic cysts of the jaws after periapical or radicular cysts, while a dentigerous cyst associated with a supernumerary tooth is a rare entity. A review of the literature since 1988 disclosed 16 reported cases of dentigerous cysts associated with premaxillay supernumerary teeth $(1,3,10-15)$. Our four cases bring the total number of documented cases to 20 (Table I). The reported prevalence of dentigerous cysts associated with supernumerary teeth ranges between 1 and $9.9 \%$ in the literature $(6,7,9,11,16)$. Most reports revealed a peak incidence of dentigerous cysts in the second and third decades of life. The age range for reported cases varies widely, from 9 to 71 years of age. The mean age of the 16 previously reported cases, not including our four cases, was 30.5 years. The mean age of the present four cases was 44.3 years. Among the 20 cases summarized in Table I, the incidence is significantly higher in men $(n=14)$ compared to women $(n=6)$. This finding is similar to previous studies, which found that dentigerous cysts appear to have a distinct predilection for the male gender $(4,17)$.

Dentigerous cysts associated with supernumerary teeth in the premaxilla are easily diagnosed radiographically due to their radiopaque image. Panoramic and upper occlusal radiographs are simple and inexpensive methods, which can be used to determine the location of dentigerous cysts, the structure of the impacted teeth, the influence on adjacent teeth and the resorption of adjacent roots (18). However, radiograph film has two inevitable disadvantages: i) a ghost image which 


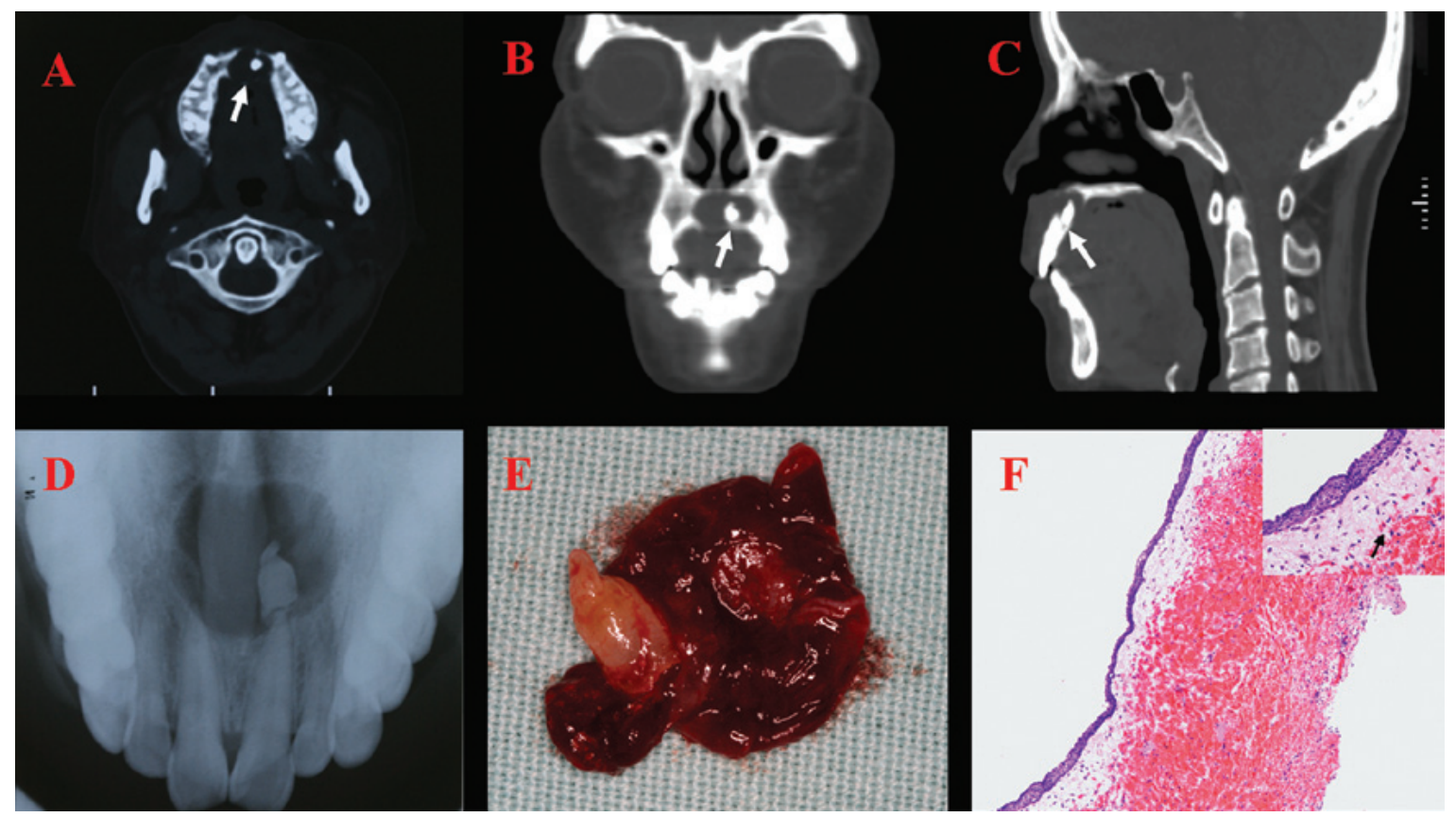

Figure 1. Case 1. (A-C) CT reveals that the cyst and the supernumerary tooth are located in the anterior maxillary region (white arrows). (A) Axial; (B) coronal; (C) sagittal planes. (D) Occlusal radiograph showing a unilocular radiolucent lesion surrounding the crown of an inverted supernumerary tooth. (E) Excised specimens show a cystic soft tissue associated with a supernumerary tooth. (F) Histopathological examination shows that the cyst was lined by non-keratinizing squamous epithelium. The connective tissue walls are cellular and composed of fibrous collagen, while some areas exhibit mononuclear inflammatory infiltration (black arrow). H\&E staining; magnification, x10.

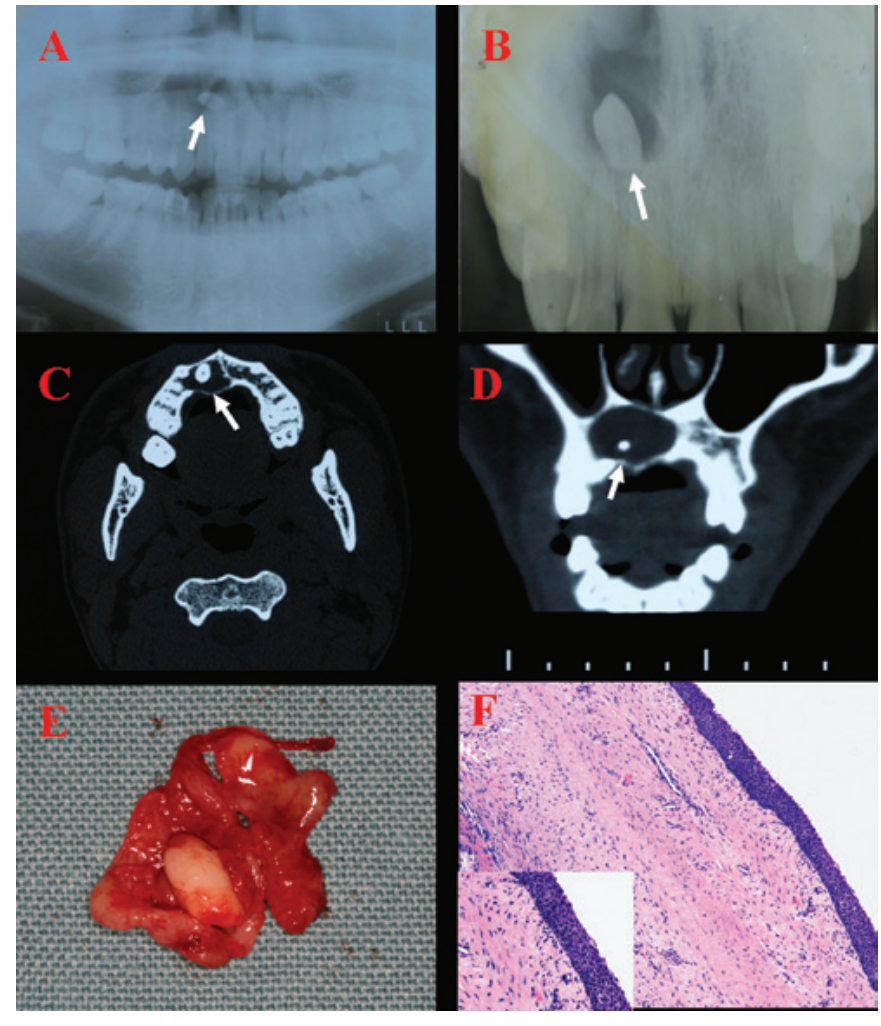

Figure 2. Case 2. (A and B) Radiological examination shows a unilocular radiolucent lesion surrounding the crown of a supernumerary tooth (arrows). (A) Panoramic radiograph; (B) occlusal radiograph. (C and D) CT reveals that the cyst and the supernumerary tooth are located in the right premaxillary region (arrows). (C) Axial; (D) coronal. (E) Excised specimens showing a cystic soft tissue associated with a supernumerary tooth. (F) Histological image indicating a cystic lining with non-keratinizing squamous epithelium. H\&E staining; magnification, $x 10$.

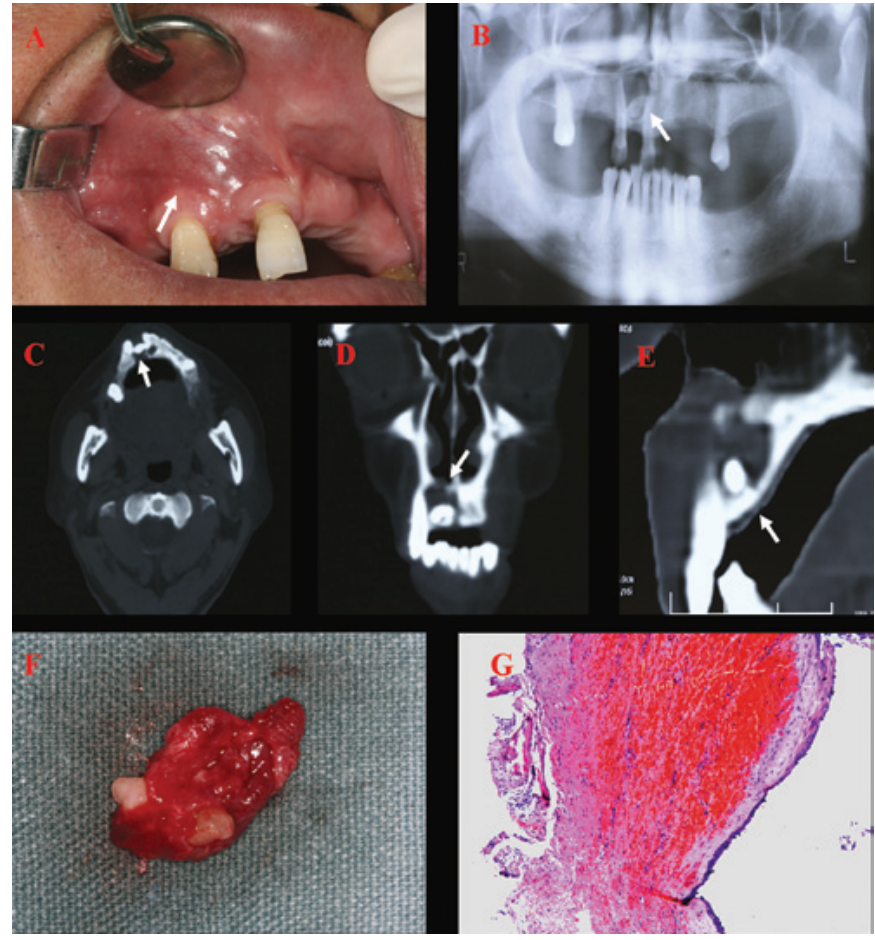

Figure 3. Case 3. (A) Intraoral view showing a labial cyst-like swelling in the anterior maxilla (arrow). (B) Panoramic radiograph shows a unilocular radiolucent lesion surrounding the crown of a supernumerary tooth between the maxillary right canine and the right central incisor (arrow). (C-E) CT reveals that the cyst and the supernumerary tooth are located in the right premaxillary region (white arrows). (A) Axial; (B) coronal; (C) sagittal planes. (F) Excised specimens show a cystic soft tissue associated with an impacted tooth. (G) Histopathological examination indicates that the cyst was lined by non-keratinizing squamous epithelium. The connective tissue walls are cellular and composed of fibrous collagen, and mononuclear inflammatory infiltration and hemorrhage are exhibited. H\&E staining; magnification, x10. 


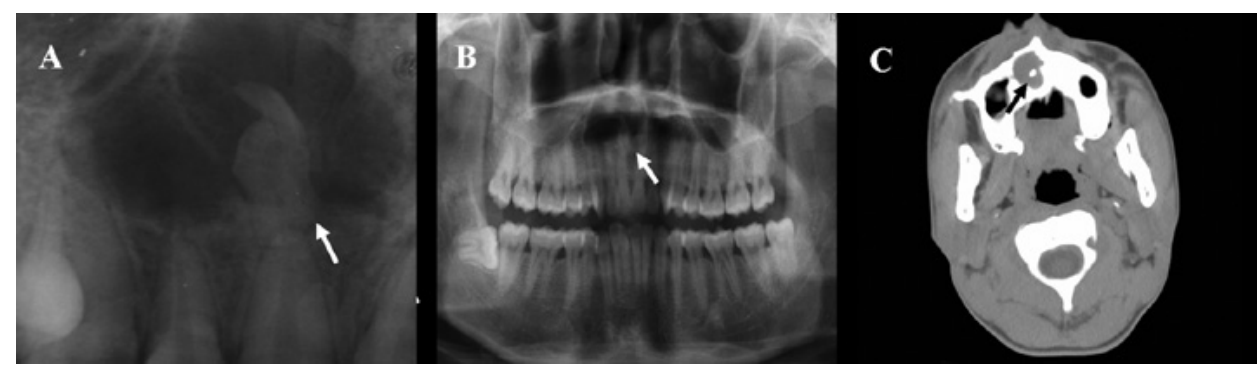

Figure 4. Case 4. (A) Occlusal radiograph shows a unilocular radiolucent lesion surrounding the crown of an inverted supernumerary tooth. (B) Panoramic radiograph shows a unilocular radiolucent lesion surrounding the crown of a supernumerary tooth extending from the maxillary right first molar to the right cental incisor. (C) Axial non-contrast CT reveals that the cyst involving an impacted tooth is located in the right premaxillary region.

Table I. Documented cases in the literature of dentigerous cysts associated with supernumerary teeth.

\begin{tabular}{|c|c|c|c|c|c|}
\hline Authors/Reference & $\begin{array}{l}\text { Patient age } \\
\text { (years) }\end{array}$ & Gender & Site (premaxilla region) & Symptoms & Treatment \\
\hline Lustmann and Bodner (3) & 9 & Female & Right central incisor & - & Enucleation \\
\hline Lustmann and Bodner (3) & 12 & Male & Left cental and lateral incisor & - & Marsupialization \\
\hline Lustmann and Bodner (3) & 37 & Male & Entire premaxilla & Swelling, pain & Enucleation \\
\hline Lustmann and Bodner (3) & 38 & Male & $\begin{array}{l}\text { Midline to the left, maxillary } \\
\text { second premolar }\end{array}$ & Swelling & Enucleation \\
\hline Lustmann and Bodner (3) & 68 & Female & $\begin{array}{l}\text { Midline to the left maxillary } \\
\text { premolar }\end{array}$ & Swelling, pain & Enucleation \\
\hline Lustmann and Bodner (3) & 71 & Female & Left premaxillary region & Asymptomatic & Enucleation \\
\hline Awang and Siar (6) & 34 & Male & $\begin{array}{l}\text { Midline to the upper left } \\
\text { first premolar }\end{array}$ & Swelling & Enucleation \\
\hline Awang and Siar (6) & 24 & Female & $\begin{array}{l}\text { From the upper right canine to } \\
\text { the upper left central incisor }\end{array}$ & Swelling & Enucleation \\
\hline Scolozzi, et al (21) & 42 & Male & Premaxilla & Swelling & $\begin{array}{l}\text { Enucleation and } \\
\text { autogenous } \\
\text { cancellous bone } \\
\text { graft from the } \\
\text { iliac crest }\end{array}$ \\
\hline Dinkar, et al (15) & 14 & Female & Maxillary anterior region & Pain, swelling & Enucleation \\
\hline Khan, et al (20) & 24 & Male & Incisor region & Swelling & Enucleation \\
\hline Gulses, et al (13) & 10 & Male & Right central incisor & Asymptomatic & Enucleation \\
\hline Kumar, et al (10) & 14 & Male & Central incisor region & Swelling & Enucleation \\
\hline John, et al (7) & 22 & Male & $\begin{array}{l}\text { From the maxillary right central } \\
\text { incisor to the right canine }\end{array}$ & Swelling & Enucleation \\
\hline John, et al (7) & 24 & Male & Maxillary anterior region & Swelling & Enucleation \\
\hline John, et al (7) & 46 & Male & Maxillary anterior region & Pain, swelling & Enucleation \\
\hline \multicolumn{6}{|l|}{ Present study } \\
\hline Case 1 & 55 & Female & Central incisors & Swelling & Enucleation \\
\hline Case 2 & 46 & Male & Midline to the upper right canine & Swelling & Enucleation \\
\hline Case 3 & 53 & Male & $\begin{array}{l}\text { From the maxillary right central } \\
\text { incisor to the right canine }\end{array}$ & Swelling & Enucleation \\
\hline Case 4 & 23 & Male & $\begin{array}{l}\text { From the maxillary right first } \\
\text { molar to right cental incisor }\end{array}$ & Swelling & - \\
\hline
\end{tabular}

cannot reflect the 3-dimensional structure of the lesion owing to a low resolution ratio, and ii) different degrees of distortion or amplification. Therefore, $\mathrm{CT}$ is necessary and valuable to obtain more information concerning the lesion. CT can be used, not only to identify the pathology of the dentigerous cyst and the exact location of the impacted tooth, but also to determine the full extent of the lesion, thus contributing to proper treatment planning as well. Meanwhile, CT can also be used to identify erosion of cortical bone and invasion into adjacent soft tissues. In the present four cases, the 
dentigerous cyst with the supernumerary tooth appeared to be within the anterior maxilla in the panoramic radiograph or/and upper occlusal radiograph. In order to determine the exact location of the impacted tooth and the degree of bone destruction, CT images were scrutinized. Therefore, to ensure appropriate treatment decisions and follow-up based on accurate information regarding dentigerous cysts associated with supernumerary teeth, we recommend panoramic radiograph and/or upper occlusal radiograph as a first-line diagnostic tools, and further evaluation of the lesion by CT examination.

Enucleation is the standard treatment for a dentigerous cyst along with extraction of the associated supernumerary tooth $(9,10)$. Among the documented cases in the literature, the second case (Table I) was treated by marsupialization because of the intimate relation of the lesion to the apices of the incisor teeth. Marsupialization is recommended for a large cyst when a single draining may not be effective and complete removal of the surrounding structure is not desirable (19). For a large cyst, Scolozzi et al recommended enucleation followed by an immediate bone grafting procedure (21). In the present four cases, surgical removal of the impacted supernumerary tooth and enucleation without using bone grafting of the associated cyst were performed.

A broad range of conditions may lead to a clinical presentation of painless swelling along the lingual surface of the palate or on the upper lip. Differential diagnosis of a median palatine cyst, nasopalatine duct cyst, radicular cyst, odontogenic keratocyst (OKC) or adenomatoid odontogenic tumor (AOT) was considered in our cases. Median palatine cysts and nasopalatine duct cysts are not associated with non-vital teeth as non-odontogenic cysts of the hard palate $(20,21)$. Most radicular cysts appear as round or pear-shaped, unilocular, lucent lesions in the periapical region, and the associated tooth usually has a deep restoration or large carious lesion radiographically (21). Approximately $40 \%$ of OKCs contain an impacted tooth, and the lumen of the cyst often contains 'cheesy' material and has a parakeratinized epithelium lining. They are more likely to show aggressive growth than other odontogenic cysts and may have undulating borders and a multilocular appearance upon radiography (22). Approximately $75 \%$ of cases are associated with an unerupted tooth, and the most common location is in the anterior maxilla. AOTs are more common in young people, affect females more than males and, most importantly, the radiolucency in cases of AOTs extends apically beyond the cementoenamel junction (18).

In conclusion, dentigerous cysts arising from impacted supernumerary teeth in the anterior maxilla should be considered in the differential diagnosis for painless swelling along the lingual surface of the palate or on the upper lip. To prevent the development of a dentigerous cyst and to avoid unwanted effects on adjacent teeth, early detection consisting of a thorough clinical and radiographical examination is necessary for accurate diagnosis and proper treatment planning.

\section{Acknowledgements}

This study was supported by grants from the Doctoral Innovation Foundations of Shanghai Jiao Tong University
School (no. BXJ 0922), and the Science and Technology Commission of Shanghai (no. 08DZ2271100).

\section{References}

1. Shah A, Gill DS, Tredwin C, et al: Diagnosis and management of supernumerary teeth. Dent Update 35: 519-520, 2008.

2. Ochsenius G, Escobar E, Godoy L, et al: Odontogenic cysts: analysis of 2,944 cases in Chile. Med Oral Patol Oral Cir Bucal 12: 85-91, 2007.

3. Lustmann J and Bodner L: Dentigerous cysts associated with supernumerary teeth. Int J Oral Maxillofac Surg 17: 100-102, 1988.

4. Arathi R and Ashwini R: Supernumerary teeth: a case report. J Indian Soc Pedod Prev Dent 23: 103-105, 2005.

5. Ikarashi T, Fujimori Y, Ohshiro K, et al: Dentigerous cyst associated with a supernumerary malformed tooth: report of a case and a clinicopathologic review. Oral Med Pathol 8: 55-59, 2003.

6. Awang MN and Siar $\mathrm{CH}$ : Dentigerous cyst due to mesiodens: report of two cases. J Ir Dent Assoc 35: 117-118, 1989.

7. John T, Guna Shekhar M, Koshy M, et al: Dentigerous cyst associated with supernumerary teeth: a report of three cases. J Clin Diagn Res 4: 2601-2606, 2010.

8. Stafne EC: Supernumerary upper central incisor. Dent Cosmos 73: 976-980, 1931.

9. Garvey MT, Barry HJ and Blake M: Supernumerary teeth - an overview of classification, diagnosis and management. J Can Dent Assoc 65: 612-616, 1999.

10. Kumar NM, Ramadevi S, Vanaki SS and Puranik RS: Dentigerous cyst occurring in maxilla associated with supernumerary tooth showing cholesterol cleft - a case report. Int J Dental Clin 2: 39, 2010.

11. Koca H, Esin A and Aycan K: Outcome of dentigerous cysts treated with marsupialization. J Clin Pediatr Dent 34: 165-168, 2009.

12. Buyukkurt MC, Omezli MM and Miloglu O: Dentigerous cyst associated with an ectopic tooth in the maxillary sinus: a report of 3 cases and review of the literature. Oral Surg Oral Med Oral Pathol Oral Radiol Endod 109: 67-71, 2010.

13. Gulses A, Karacayli U and Koymen R: Dentigerous cyst associated with inverted and fused supernumerary teeth in a child: a case report. OHDMBSC 1: 38-41, 2009.

14. Karaçal N, Ambarcoğlu O and Kutlu N: Median palatine cyst: report of an unusual entity. Plast Reconstr Surg 115: 1213-1214, 2005.

15. Dinkar AD, Dawasaz AA and Shenoy S: Dentigerous cyst associated with multiple mesiodens: a case report. J Indian Soc Pedod Prev Dent 25: 56-59, 2007.

16. Righini CA, Boubagra K, Bettega G, et al: Nasopalatine canal cyst: 4 cases and a review of the literature. Ann Otolaryngol Chir Cervicofac 121: 115-119, 2004

17. Avelar RL, Antunes AA, Carvalho RW, et al: Odontogenic cysts: a clinicopathological study of 507 cases. J Oral Sci 51: 581-586, 2009.

18. Wang CJ, Huang PH, Wang YL, et al: Dentigerous cyst over maxillary sinus: a case report and literature review. Taiwan J Oral Maxillofac Surg 20: 116-124, 2009.

19. Giancotti A, Grazzini F, De Dominicis F, et al: Multidisciplinary evaluation and clinical management of mesiodens. J Clin Pediatr Dent 26: 233-237, 2002.

20. Khan MH, Alam MT, Haque S, et al: Upper lip swelling caused by a large dentigerous cyst with mesiodens. Mymensingh Med J 17: S100-S103, 2008

21. Scolozzi P, Lombardi T and Richter M: Upper lip swelling caused by a large dentigerous cyst. Eur Arch Otorhinolaryngol 262: 246-249, 2005.

22. Zhang LL, Yang R, Zhang L, et al: Dentigerous cyst: a retrospective clinicopathological analysis of 2082 dentigerous cysts in British Columbia, Canada. Int J Oral Maxillofac Surg 39: 878-882, 2010. 